\title{
Gestión Pública y salud: el caso de la Leishmaniasis en el estado Sucre
}

\author{
Betancourt, Belkis* \\ Alarcón, Luis** \\ Gómez, Irey***
}

\section{Resumen}

El objetivo de este trabajo es analizar el fenómeno salud-enfermedad, haciendo énfasis en la presencia de la leishmaniasis y la percepción que tienen los pacientes y no pacientes con respecto a esta enfermedad y al servicio que prestan los organismos de salud, en la atención, prevención y control de la misma. El referente empírico lo constituyen los habitantes de las comunidades de San Pedrito, San Pedro y Vega Grande, ubicadas en el municipio Sucre, del estado Sucre, las cuales hacen uso del Servicio de Dermatología Sanitaria (SDS), en la ciudad de Cumaná. Se utilizó la entrevista estructurada y la observación participante. Entre los resultados cabe destacar que el estilo de vida de los pacientes y el desconocimiento acerca de la leishmaniasis inciden en su prevalencia y emergencia. De igual modo, la percepción y los prejuicios de los pacientes inhibe el uso de los centros de salud y la puesta en práctica de medidas preventivas. El rol del SDS es principalmente curativo, siendo los pacientes y la comunidad en general, sujetos pasivos en el proceso de tratamiento y control de la enfermedad. Se concluye que para mejorar la gestión es conveniente descentralizar el SDS y considerar las características socioeconómicas y socioculturales de los usuarios.

Palabras clave: Leishmaniasis, salud, gestión pública.

Recibido: 04-06-29. Aceptado: 04-08-27

* $\quad$ Asistente de investigación del grupo de Investigaciones e Innovaciones Sociales SOPHIS, en el proyecto de salud ejecutado actualmente con el apoyo del FONACIT y el Consejo de Investigación de la Universidad de Oriente (UDO).

** Sociólogo y Lic. en Educación. Participante en el postgrado de Ciencias Políticas de la Universidad Simón Bolívar (USB). Investigador del Grupo SOPHIS y de la USB. Docente de la Universidad Central de Venezuela (UCV). Adscrito al PPI del FONACIT.

*** Trabajadora Social. Magister en Planificación del Desarrollo Regional. Aspirante al Doctor en Ciencias Sociales en la UCV. Profesora asociada, investigadora-Coordinadora del grupo de Investigaciones e Innovaciones Sociales-SOPHIS de la UDO y responsable del proyecto de investigación en salud, financiado por el FONACIT, con el apoyo del Consejo de Investigación de la UDO. Adscrita al PPI DEL FONACIT. 


\section{Public Management and Health: the Case of Leishmaniasis in Sucre State}

\section{Abstract}

The objective of this paper is to analyze the health-illness phenomenon, placing emphasis on the presence of leishmaniasis and the perception that patients and non-patients have in relation to this disease, as well as the services offered by health organisms, and the attention, prevention and control of the same. The empirical reference source is made up of the inhabitants of the San Pedrito, San Pedro, and Vega Grande townships located in Sucre Municipality, Sucre State. All of these inhabitants utilize the Dermatalogical Sanitary Service (SDS) in Cumana city. A structured interview was applied as well as participant observation. Among the results it is important to point out the life styles of the patients, and the lack of knowledge that exists in reference to the incidence of leishmaniasis and its prevalence and emergency status. In a similar manner the perceptions and prejudices of the patients inhibit their use of health centers and the effectiveness of preventive measures. The role of SDS is basically curative, and the patients from these communities are passive subjects in the treatment and control process. The conclusion is that to improve the management of this problem it would be convenient to de-centralize SDS and consider the socio-economic and socio-cultural characteristics of the users.

Key words: Leishmaniasis, health, public management.

\section{Introducción}

La Leishmaniasis es una zoonosis endémica producida por distintas especies de protozoarios pertenecientes al género Leishmania. Se presenta como infección oportunista en pacientes inmunodeprimidos (bajas defensas), suele afectar a niños, jóvenes y adultos. Generalmente, esta enfermedad es más común en zonas rurales, selváticas y boscosas que en áreas urbanas; sin embargo, puede encontrarse en las cercanías de ciertas ciudades. El riesgo de contraer la endemia es más alto para las personas que se encuentran al aire libre en zonas endémicas de Leishmaniasis entre la oscuridad y el amanecer. Algunas causas de la patología radican fundamentalmente en la pobreza generalizada, pésimas condiciones sanitarias, carencias de servicios públicos y las influencias climáticas.
Esta enfermedad tropical, según la Organización Mundial de la Salud (OMS, 2002) ha afectado aproximadamente a 12 millones de personas repartidas en 88 países tropicales y subtropicales alrededor del mundo. Epidemiológicamente, la Leishmaniasis por su alta incidencia se ha convertido en un grave problema de salud pública, a nivel mundial. Específicamente, en Venezuela donde dicha enfermedad ha adoptado un carácter emergente y reemergente como consecuencia del profundo deterioro social, caracterizado por aspectos, tales como, el incremento de los índices de pobreza, la merma de la calidad de vida y el descenso considerable del nivel de vida de la población. Por otra parte, los servicios de salud, también se han visto afectados debido a que las instituciones públicas prestadoras de éste servicio en su mayoría no satisfacen 
las demandas sanitarias de la colectividad por carecer del personal calificado, recursos materiales y reducción o insuficiencia presupuestaria, además, de la rigidez e inflexibilidad de esquemas operativos y organizativos heredados de gestiones del servicio de salud basadas en paradigmas conservadores y burocráticos.

A lo anterior se agregan otros aspectos de carácter socio-ambiental y socio-cultural que también están contribuyendo al deterioro de los indicadores de salud y a una regresión en el perfil epidemiológico. Específicamente, en el caso de la leishmaniasis en el estado Sucre, esto se expresa en la prevalencia y resurgimiento de la enfermedad, con focos de alta y baja incidencia en varias áreas endémicas, económicamente deprimidas de esta región, convirtiéndose así en una problemática de salud, ya que la enfermedad emerge progresivamente con importantes implicaciones clínicas, epidemiológicas, económicas y sociales. De ahí la necesidad de explorar este tema, considerando esta vez la óptica de aquellos que de forma directa o indirecta han sido afectados por esta endemia.

Este trabajo constituye un avance de una investigación cualitativa de carác- ter más amplio en la cual se consideran otras variables relativas al proceso salud-enfermedad (representaciones sociales y estilo de vida de los pacientes y prestadores del servicio, gestión del servicio y participación comunitaria). El eje central de la investigación lo constituye la perspectiva de los sujetos involucrados en la atención, control y prevención de la leishmaniasis (pacientes, comunidad y prestadores del servicio).

En cuanto a la metodología, para la realización de este estudio se diseñó una estrategia que consistió primeramente en la realización de un conjunto de actividades destinadas a la inserción en el área de estudio (dependencias de salud y comunidades). Paralelamente, se hizo una revisión de los registros de casos que con respecto a la leishmaniasis llevan a cabo los organismos competentes. El período revisado abarcó de 1998 al 2002. Una vez avanzada esta etapa y considerados los informes epidemiológicos, boletines, fichas médicas y otras fuentes, se procedió a escoger una muestra, tomando en cuenta criterios epidemiológicos y sociológicos (aspectos psicosociales, sociodemográficos y de carácter tempo-espacial). Dicha muestra estuvo integrada por pacientes infectados ${ }^{1}$, no pacientes ${ }^{2}$ e in-

1 Pacientes infectados: son todas aquellas personas que presentan lesiones Leishmánicas o han padecido la Leishmaniasis y se clasifican en: a) Pacientes activos: Son todos los pacientes tratados y cuya lesión aún presenta signos de infiltración, descamación, adenopatías o lesiones satélites (lesión no curada); b) Posterior de alta: Corresponde a los pacientes que han curado su lesión clínicamente y se mantiene en observación; c) Perdidos: Cuando el paciente no acude a su control por un lapso mayor a los seis (6) meses.

2 No Pacientes: están constituidos por los familiares más cercanos de los pacientes (padres, hermanos, hijos, cónyuges así como los amigos, vecinos y demás personas), que comparten y tienen cierto acercamiento con el enfermo. 
formantes clave ${ }^{3}$, que habitan en las comunidades de San Pedro, San Pedrito y Vega Grande del municipio Sucre en el estado Sucre. En estas comunidades se ha presentado un importante número de personas afectadas por esta enfermedad, atendidas por el Servicio de Dermatología Sanitaria (SDS) ubicado en el Ambulatorio "Arquímedes Fuentes" de la ciudad de Cumaná. Estos constituyen los referentes empíricos de esta investigación. Para la recolección de la información se utilizaron técnicas, tales como, revisión documental, observación participante y no participante, entrevistas y coloquios familiares. Los datos fueron registrados en diarios de campo, guías de cuestionario, grabadoras y video-grabadoras.

El objetivo de este trabajo es analizar el fenómeno salud-enfermedad, haciendo énfasis en la presencia de la Leishmaniasis y la percepción que tienen los pacientes y no pacientes con respecto a la misma, así como al servicio que prestan los organismos de salud, en el proceso de atención, prevención y control. Se parte de la premisa acerca de la importancia que tiene el conocimiento de la racionalidad de los actores que participan en este proceso, y las ventajas que esto puede brindar en el diseño y ejecución de políticas sanitarias que atiendan efectivamente este tipo de enfermedades.

\section{Consideraciones generales}

A continuación se exponen algunas referencias de carácter teórico que apoyan el tema en estudio. La salud está estrechamente vinculada a la vida cotidiana de los sujetos y poblaciones, porque ésta constituye el tejido social de las relaciones e interacciones sociales, que son básicamente comunitarias y predominantemente societarias. En tal sentido, la familia como institución social tiene una influencia tremenda sobre cada uno de sus miembros y en alta medida el comportamiento en torno a la salud está vinculado a las representaciones sociales, que ellos tienen con respecto a la salud y a las enfermedades. Siendo las representaciones sociales "un conjunto de conceptos, declaraciones y explicaciones originadas en la vida cotidiana, en el curso de las comunicaciones interindividuales". Estas también equivalen en "nuestra sociedad, a los mitos y sistemas de creencias de las sociedades tradicionales; incluso puede afirmarse que son la versión del sentido común" (Moscovici, 1961:181). Las formas de pensar y crear la realidad van a estar constituidas por elementos de carácter simbólico, ya que no solamente son formas de adquirir y reproducir el conocimiento, sino que tienen la capacidad de dotar de sentido a la realidad social, pues, su finalidad es transformar lo desconocido en algo familiar.

3 Informantes Clave: Son referentes empíricos de gran importancia para la adquisición de información, entre estos tenemos: el personal técnico-operativo adscrito al Servicio de Dermatología Sanitaria (S.D.S) y a FUNDASALUD (médicos, enfermeras, inspectores sanitarios y promotores sociales, y finalmente aquellas personas denominados curanderos, hierbateros o botánicos, que son también habitantes de las localidades estudiadas. 
Teóricamente (en este caso, en su relación con la salud), la representación social involucra ciertas dimensiones: información, conocimiento tradicional y popular de la gente, sus creencias y la imagen que se construye acerca de lo qué es la enfermedad y lo que representa para los sujetos. Además, de los distintos elementos que van a tener implicaciones prácticas que dan cuenta del significado que tienen para ellos la salud, cómo se conserva, cómo se pierde, la explicación sobre las distintas enfermedades, sobre su tratamiento y la idea que tienen acerca de las formas de gestión de los servicios médicos y las instituciones de salud pública en general. De igual manera, la responsabilidad de enfermarse y consecuentemente lo que hacen para curarse.

En general, las representaciones sociales no sólo son productos mentales sino que son construcciones simbólicas que se crean y recrean en el curso de las interacciones sociales, que están relacionadas con la realidad social y con la racionalidad popular de los actores sociales. Por esa razón, tienen un carácter eminentemente dinámico y están constantemente en actividad de construcción y reconstrucción. En esa realidad el conocimiento se construye, reconstruye y circula como una evidencia armónica, independiente de los hombres y las respectivas situaciones que dan cavidad a la existencia y reproducción de la vida cotidiana. Conocimiento que surge de la situación cara a cara no solamente con sus semejantes, con quienes mantiene una interacción directa sino con sus contemporáneos, sus antecesores y sucesores. Siendo el lenguaje el principal sistema simbólico que hace posible por excelencia la acumulación o acopio social del conocimiento, el cual como dicen Berger y Luckman (1994:40) "se transmite de generación en generación y está al alcance del individuo en la vida cotidiana..."

Con respecto al proceso salud enfermedad, se plantea que las representaciones sociales tienen influencia en el comportamiento social en un contexto social determinado. Sobre este punto se han señalado tres dimensiones fundamentales de las representaciones sociales inherentes al fenómeno salud - enfermedad (Kornblit, 2000), las cuales son:

Individuo-sociedad: dimensión que deja ver el conflicto entre el individuo y la sociedad. En ella la enfermedad es concebida como causalidad externa y se le niega responsabilidad al sujeto sobre su salud.

Conocimiento tradicional y folklóri$c o$ : en ésta se concretiza el pensamiento social y se elaboran símbolos que son socialmente compartidos, por los sujetos que orientan las acciones sociales y la conducta colectiva.

Origen de la enfermedad:ésta busca las causas que el sujeto argumenta ante su responsabilidad por haberse enfermado $y$ a su vez lo que hacen para curarse.

El conocimiento de las representaciones de la salud y la enfermedad en relación a la medicina y el saber médico también ofrece las posibilidades de elección en relación con la medicina (la tradicional o la alternativa), y la condición de paciente activo en correspondencia con la experiencia en la aplicación de un tratamiento (por el hecho de vivir con la enfermedad y no ser testigos externos a ella como los médicos). Además, son importantes las opiniones que tienen los pacientes sobre el personal médico y las 
instituciones de salud a fin de determinar la eficacia del tratamiento, las medidas de prevención y control, y otras acciones terapéuticas.

Otro aspecto relevante es la responsabilidad de transformar positivamente la salud, y el papel que en estos juegan los sujetos, ya que su estilo de vida puede constituirse en un factor de riesgo en la medida que las condiciones socio-económicas, socio-culturales y socio-ambientales a ella asociadas no sean las más apropiadas o que simplemente se encuentren ausentes de ese entorno social. El estilo de vida en un sentido positivo también puede potenciar el bienestar colectivo y por ende la salud. La salud se relaciona con la vida cotidiana, la primera es parte de cada grupo humano y de cada persona y la segunda es a su vez, "un instante del proyecto de vida, conciente o no, de dicho grupo y personas como sujetos sociales, como actores" (Martínez et al, 1998: 85).

En el estudio del tema de salud confluyen lo biológico, lo social, el sujeto y la comunidad. Existe una visión de la salud como el completo estado de bienestar biopsicosocial que puede alcanzarse solamente cuando las personas libres y en armonía con su ambiente interaccionan con otros seres humanos. De esa manera, la salud estará dada en función de la relación que el hombre establezca con su medio ambiente y demás sujetos sociales. Por consiguiente, toda enfermedad constituye un fenómeno social con orígenes en factores sociales de riesgo que influyen en la evolución y consecuencias de la misma, no sólo para el enfermo sino para toda la sociedad. En este sentido, la salud-enfermedad "... no son dos estados opuestos que se presen- tan en la vida del hombre, sino una variación del proceso de adaptación del organismo al medio en que se sirve" (Aranda, 1991:55). De modo tal que el ambiente puede influir (su acción puede ser de diversa naturaleza: física, biológica, psicológica, económica, social o cultural), actuando constantemente o permanentemente sobre los sujetos unas veces de manera beneficiosa y otras perjudiciales.

En ese sentido, en lo concerniente al aspecto sanitario se encuentran involucrados, al menos, dos actores sociales relevantes: el gobierno, representado por las instituciones de salud pública, a quien le corresponde la formulación y ejecución de políticas y programas de salud a fin de prever y controlar las endemias. El otro actor es la población o los usuarios quienes de manera activa o potencial legitiman la acción gubernamental en salud, debiendo asumir un rol protagónico en el sistema de salud.

Con respecto a las instituciones públicas de salud, teóricamente éstas llevan a cabo un proceso que implica acciones y toma de decisiones relativas a garantizar el mantenimiento y preservación de la salud y por ende de la calidad de vida de la población. La gestión de los organismos de salud se expresa en un conjunto de actividades científico-técnicas o científico-operacionales que buscan atribuir valor de eficiencia y eficacia a los procesos de intervención, diagnóstico y en la aplicación del tratamiento o vacunación, así como otros modos terapéuticos en el control, prevención y merma de las enfermedades.

Por su parte, la población o usuarios, para tener ese rol protagónico deben poseer un grado de responsabilidad en la gestión de la salud, que posibilite su parti- 
cipación en la toma de decisiones destinadas a la atención, prevención y control sanitario. En este particular pueden contribuir modelos de participación que rompan con el esquema pasivo y generen una participación orgánica con nuevas formas de liderazgo y de toma de decisiones. No hay que perder de vista que la participación y el compromiso de la gente puede facilitarse mediante la vinculación con su vida cotidiana. Pero esto es posible en la medida que los programas sociales logren articular asertivamente los diversos y contradictorios intereses de beneficiarios y prestadores de servicios. Ello conduce inevitablemente al replanteamiento de la visión tradicional de la gerencia social, que no estaría ya centrada exclusivamente en intereses particulares y en una concepción corporativa sino inscrita en un proyecto social, un proyecto colectivo. En sentido teórico, la gerencia social es la negociación de la gestión administrativa para producir mayor efectividad programática al utilizar los recursos comunales e institucionales más racionalmente. En la gerencia social, no hay subordinados sino colaboradores, y la negociación es básica para establecer la red de contactos entre actores. De igual modo, la gerencia social es definida como "una actividad de dirección y organización de personas para el logro de los objetivos que una organización se ha propuesto, haciendo un uso racional (eficiente y eficaz) de los recursos" (Navarro citado por Molina y Morera, 1999). Sin em- bargo, la gerencia de lo social es un asunto bastante complejo por la dimensión y amplitud de la cuestión social, relacionada con el bienestar social y más concretamente con el desafío que representa la satisfacción de necesidades sociales.

De este modo, la participación y la organización social tienen que ser vistas como los espacios idóneos y necesarios para lograr la educación, formación y movilización de la gente, con el propósito de captar las demandas reales y vincularlas directamente a procesos democráticos de toma de decisión en la etapa de diseño, ejecución y evaluación de las políticas de salud.

Cabe destacar que una de las desventajas que caracterizan al servicio de salud es la complejidad que representa la administración de este sector, caracterizado, entre otras cosas, por presentar una “... organización programática que disminuye la capacidad de lograr los resultados con eficiencia, efectividad y ahorro de recursos, porque existe una brecha del retraso de la funcionalidad estadal y el movilismo de las operaciones no estadales que implica un desperdicio de factores y medios instrumentales no mensurados..." (Graterol et al, 1998).

\section{El Servicio de Dermatología Sanitaria (SDS) y la atención de la Leishmaniasis}

En el estado Sucre, existe un elevado número de pacientes con Leishmaniasis Cutánea ${ }^{4}(L, C)$, en proceso de con- 
trol y tratamiento, lo que hace prever el riesgo de contraer la enfermedad, ya que sólo se actúa en el último eslabón de la cadena epidemiológica: tratamiento y rehabilitación. Para el período 19881990, según registros del Departamento de Dermatología Sanitaria, la región ocupó el séptimo lugar a nivel nacional con 156 casos de este tipo de endemia. Actualmente la Leishmaniasis según registros del Instituto de Biomedicina de la Universidad Central de Venezuela (UCV), reporta una incidencia de 4000 casos anuales en Venezuela. Específicamente, las entidades federales con mayor prevalencia de esta enfermedad son: Lara, que sigue ocupando el primer lugar con 398 casos; Mérida en segundo lugar con $233 \mathrm{ca}$ sos; Miranda ocupando el tercer lugar con 218 casos y el estado Sucre con las cifras antes indicadas.

De acuerdo a los registros de los organismos sanitarios, las áreas geográficas más afectadas por LC en la región sucrense corresponden a la Península de Paria (Municipios Bermúdez, Arismendi y Andrés Mata) que, en líneas generales, representan un $80 \%$ de la ocurrencia anual de los casos reportados. A ello se agrega el repunte e incremento de la Leishmaniasis en el municipio Sucre y otras áreas del municipio Montes (M.S.D.S.; Coordinación Regional de
Dermatología Sanitaria, s/f). Es por ello que el S.D.S. desempeña un rol muy importante en lo que concierne a esta enfermedad. Este Servicio funciona en las ciudades de Carúpano y Cumaná, abarcando toda la región sucrense, en el suministro de atención, tratamiento y control a los pacientes de Leishmaniasis.

En esta investigación hemos tomado como referencia la sede ubicada en el Ambulatorio "Arquímedes Fuentes" de Cumaná, en la cual reciben atención pacientes que proceden de las diferentes localidades que conforman los municipios Sucre y Montes de esta región. EI S.D.S está estructurado por el siguiente personal administrativo y técnico-operativo: un Coordinador de la Unidad Programática de Dermatología Sanitaria, un médico adjunto al Servicio, un Inspector Sanitario y una secretaria. Además, de un grupo de Promotores Sociales de FUNDASALUD que en las localidades hacen su respectivo trabajo con los pacientes.

EI SDS es supervisado por el Instituto de Biomedicina de la UCV, realizando consultas de control regular de la Leishmaniasis. Allí se efectúa una evaluación médica al paciente y cuando exista la sospecha de la endemia, el inspector sanitario procede a realizarle lo que desde el punto de vista médico se denomina un frotis directo ${ }^{5}$ en las lesiones ulcera-

pápulas eritematosas que aumentan de tamaño de manera lenta y progresiva, generalmente sin síntomas, auque a veces con purito (Luna, 2001).

5 Técnicas de Frotis: El examen de los frotis de tejido sencillo y de bajo costo, pero de fusibilidad pobre sobre todo en los casos de Leishmaniasis mucosa cutánea, consiste en la observación directa de los parásitos en frotis por oposición, de cultivos del parásito, histología de lesiones, identificación y taxonomía (Luna, 2001). 
das y cultivos a través del aislamiento e identificación de los parásitos. De este modo, se lleva cabo el llamado diagnóstico acertado ${ }^{6}$. Posteriormente, según los resultados se comienza con el tratamiento o Inmunoterapia Combinada ${ }^{7}$, la cual consiste en la aplicación de tres vacunas combinadas, divididas cada una en dosis aplicadas al paciente en los brazos, estás se efectuaran de tres en tres semanas o según lo facultado por el médico. Dicha terapia se efectúa previa cita de acuerdo a las semanas epidemiológicas. Además, en las consultas el médico les explica a los usuarios del servicio lo qué es la enfermedad, sus síntomas, etiología y modos de prevenirla. De igual modo, el personal técnico-operativo del SDS también se dirige en ocasiones a diferentes comunidades apartadas y ubicadas en las zonas endémicas de los municipios Sucre y Montes, para efectuar operativos que les permitan evaluar a los enfermos y hacerles los respectivos frotis y si es necesario referirlos al Servicio. Todo esto como parte de la gestión con el fin de cumplir con el diagnóstico, tratamiento, control y erradicación de la endemia. Vale destacar, que algunos pacientes viven en zonas endémicas distantes de la ciudad de Cumaná y además no cuentan con los recursos económicos y los medios de transporte necesarios para trasladarse a la consulta que ofrece el SDS. Esto trae como consecuencia que no acudan al tratamiento o deserten antes de haber culminado el mismo.

Por esa razón, la Coordinación de Dermatología Sanitaria ha insistido en la idea de lo que ellos denominan la "descentralización del SDS". De ahí que se haya planteado una estrategia que permita abordar el problema con mayor prontitud en las zonas endémicas, esta consiste en un "Modelo de Atención a nivel municipal que permita atender oportunamente a los afectados por esa enfermedad en cada municipio del estado, este modelo tendrá como base fundamental la capacitación de los equipos municipales y locales de salud para que asuman, desde sus centros de salud todo lo relacionado al diagnóstico, tratamiento, control y prevención de la Leishmaniasis, lo que coadyuva a disminuir los gastos económicos que acarrea el traslado del paciente a los servicios regionales de Dermatología Sanitaria (Cumaná-Carúpano)" (M.S.D.S., Coordinación Regional de Dermatología Sanitaria, s/f). Sin embargo, esta propuesta no se ha podido materializar, aun cuando ya se había comenzado a preparar el personal médico destinado a prestar la asistencia correspon-

6 Diagnóstico acertado de la Leishmaniasi, se basa fundamentalmente en la información procedente de la evaluación clínica, epidemiológica, inmunológica y psicológica, donde los tres primeros dan una sospecha diagnóstica y el último establece el diagnóstico definitivo de la enfermedad.

7 Tratamiento Inmunoterapia Combinada, la cual consiste en una vacuna combinada, creada por el Dr. Jacinto Convit, mezcla de Promastigostes de Leishmaniasis Mexicana, Amazonensis (PIm), muertos por autoclavado $\left(120^{\circ} \mathrm{C}\right.$ durante 20 minutos) y BCG. La concentración de PIm es de $6,4 \times 10,8$ parásitos por dosis en un volumen de $0,4 \mathrm{ml}$. 
diente. Las limitaciones que se han presentado están relacionadas con la presencia de ciertos intereses opuestos y por algunas trabas burocráticas.

\section{a) En cuanto a la Atención que presta el SDS}

Los pacientes y no pacientes opinan que la atención es buena, por cuanto reciben la asistencia médica correspondiente que los alivia de los síntomas y padecimientos de la enfermedad (fiebre, dolores, ulceraciones, malestares generales...), y que la mayoría de las veces no los dejan cumplir con las actividades domésticas y de trabajo de manera normal. En ese sentido, han manifestado que el personal prestador del servicio en el S.D.S., realizan su trabajo con responsabilidad y de un modo muy eficaz. Ellos le dan mucha importancia al hecho de que los funcionarios se dirijan a las diferentes comunidades de los municipios, haciéndoles evaluaciones a los pacientes, tomándoles el frotis y orientándolos para que éstos acudan a las consultas que ofrece el servicio. De igual forma, expresaron que para ellos es importante que tanto el médico como el resto del personal les brinde orientación e información acerca de lo que ellos deben hacer en sus respectivos hogares para limpiarse o curarse las ulceraciones leishmanicas. Además, de los modos preventivos que deben asumir con el fin de no contraer la endemia.

Otro aspecto de interés en relación a la atención, es el sentimiento de admiración, respeto y agradecimiento que regularmente los usuarios manifiestan hacia el personal de salud. Ellos opinan que no solamente fueron curados de la enfermedad sino que recibieron un trato humano de parte de los funcionarios, ya que siempre trataron de ayudarlos para que acudieran al tratamiento, especialmente en la solución del problema de transporte, pues, aunque el servicio no cuenta con estas unidades, este personal se las ingenia para trasladarlos a las consultas, haciendo gestiones que a veces van más allá de sus competencias. Esta demostración de solidaridad ha hecho que los usuarios proyecten en sus respectivas comunidades, una imagen positiva del Servicio, sirviendo esto de elemento motivador hacia aquellos pacientes que inicialmente se mostraban renuentes a recibir el tratamiento.

Por su parte, el personal que labora en el SDS opina que en líneas generales el servicio de salud es bueno, porque se está cumpliendo con la demanda de los pacientes que a diario acuden a las consultas para mejorar su estado de salud e incorporarse activamente a sus actividades cotidianas. Ellos argumentan que realizan un trabajo de equipo, con responsabilidad, ética, vocación y mística de trabajo. Por tal motivo, se sentían satisfechos de los resultados que se habían obtenido en los últimos años con respecto al incremento y repunte en la enfermedad en comparación con tres años atrás, lo cual a su juicio tiene que ver con el hecho que los habitantes de las zonas endémicas ahora manejan un poco más de información con respecto a la enfermedad, las formas de prevención y sobre todo lo concerniente a las consultas del servicio. En esto último, juega un papel importante la labor de las promotoras sociales, adscritas a este servicio. No obstante, también 
reconocen que muchos de los pacientes y no pacientes aún desconocen los modos de prevención de la endemia, lo que trae como consecuencia que se vuelvan renuentes a ir a las consultas y sobre todo a recibir tratamiento. Cabe destacar que aunque los funcionarios hacen una valoración positiva de su trabajo, también opinan que pueden ser más efectivos si se subsanan los problemas ligados a la falta de recursos materiales, uno de los más importantes es la falta de transporte. De igual manera, tienen la expectativa de una mayor participación de los pacientes en programas comunitarios destinados al conocimiento y prevención de la endemia y en el proceso mismo de atención que ellos llevan a cabo.

\section{b) En cuanto al Tratamiento}

Los usuarios del servicio y sus familiares han manifestado que aunque el tratamiento es un poco incómodo, debido a los efectos colaterales, que éste les produce (erupción, picazón e hinchazón en la zona donde le aplican las vacunas) es de gran importancia recibirlo ya que de este modo, ellos pueden curarse de la enfermedad y además, quedar inmunes para así evitar el riesgo de contraer nuevamente la enfermedad. De igual modo, expresaron que a ellos les había ido muy bien con el tratamiento, por esa razón invitaban a sus vecinos, sobre los cuales tenían sospechas que podían tener la enfermedad para que se sometieran a la prueba y posteriormente a la terapia de vacunación, y así lograrán su curación, para así erradicar el temor sobre la enfermedad, cuando éstos pensaban que podían ser un cáncer, brujería o un "daño".
Se planteó el problema del traslado de los pacientes de sus respectivas comunidades, las cuales en su mayoría se encontraban en áreas alejadas en las montañas, sin estructuras viales; así como el hecho de no contar con recursos económicos que facilitaran el traslado hacia el centro dispensador de salud. Otra razón que justifica que el tratamiento se lleve a cabo en los centros de salud más cercanos a las comunidades donde viven los pacientes es que la terapia se prepara en función de una lista de pacientes cuya asistencia ha sido previamente planificada por el SDS. Por ende, el riesgo de llegar tarde a la consulta, implica la pérdida del proceso de vacunación con todas las implicaciones negativas que esto trae para la erradicación de la enfermedad.

La Inmunoterapia Combinada o tratamiento según el personal de salud es efectiva ya que tiene un carácter inmunizador una vez aplicado al paciente. De igual modo, expresaron que la terapia de vacunación se está aplicando de manera normal, según las jornadas que tocan en las respectivas semanas epidemiológicas. Sin embargo, también dijeron que en ocasiones se presentan algunos inconvenientes cuando se terminan la medicina suministrada en el tratamiento. Vale decir al respecto, que las vacunas son fabricadas en multidosis por el Instituto de Biomedicina de la Universidad Central de Venezuela (UCV). Por lo tanto, un frasco contentivo de esta vacuna no se puede destapar para administrarle tratamiento a un solo paciente, porque ésta tiene una duración de pocas horas y ante los costos que ello implica no se puede correr el riesgo de desechar el resto del contenido del medicamento. 
De la misma manera, el personal que trabaja en el servicio manifestó la necesidad de descentralizar el tratamiento de Leishmaniasis, coincidiendo de esta forma con las opiniones de los pacientes. Expresando que la aplicación de la Inmunoterapia Combinada debía ser descentralizada para que se hiciera más accesible a los pacientes, ya que así, éstos tendrían mayor motivación y garantía de recibir el servicio. Al tiempo, que la endemia sería atendida de una manera más eficaz, el tratamiento tendría mayor viabilidad y efectividad al ser aplicado; pues, se eliminarían diversos obstáculos que no permiten que todos los pacientes puedan tener la misma oportunidad de recibir el tratamiento. De igual manera, se piensa que ésta sería una de las formas para erradicar la Leishmaniasis en las zonas endémicas de una manera más rápida. Sin embargo, aún cuando la Coordinación Regional de Dermatología Sanitaria tomó en cuenta algunas estrategias referidas a un proyecto para la descentralización del tratamiento por municipio, éste no se lleva a cabo aún al parecer por desacuerdos relacionados con diferencias de intereses y trámites burocráticos, en las instancias competentes.

\section{c) En cuanto a la prevención y control}

Con relación a este punto, algunos de los pacientes manifestaron que desconocían las formas preventivas de la enfermedad. Sin embargo, se observó que cubrían su cuerpo con pantalones largos y camisas manga larga cuando se dirigían al conuco. Por esa razón, se les preguntó ¿por qué se ponían ropas largas cuando iban al conuco? A lo que ellos contestaron: - ique era por razones de seguridad, ya que así no les picarían los mosquitos que producían las llagas! - esto se lo habían dicho las promotoras sociales, las cuales continuamente los visitaban!. Entre tanto otros usuarios expresaron que cuando ellos acudían al Servicio de Dermatología su personal les explicaba lo que era la enfermedad, su etiología y los modos preventivos; además, les daban las respectivas orientaciones y recomendaciones sobre las formas de hacerse la asepsia de las lesiones o úlceras. De la misma forma, estos pacientes expresaron que los promotores sociales también hacían en sus comunidades lo propio para informar a las personas sobre este punto.

No obstante, ellos pensaban que todavía había mucha desinformación con respecto a la enfermedad; de allí que algunos pacientes especulaban creyendo que la Leishmaniasis era una brujería, un "daño" o un castigo divino, volviéndose renuente a asistir a la consulta y que otros que aun cuando ya habían acudido a ella se volvieran renuentes al tratamiento y desertaran. Por esa razón, consideraron que era obligatorio que se hicieran campañas informativas sobre la enfermedad.

Los miembros del equipo de salud manifestaron que ellos con relación al control de la enfermedad se dedicaban principalmente a colocar el tratamiento a los pacientes que asistían a las consultas regulares para su respectivo control, el cual consistía en la terapia de vacunación. Sin embargo, también aclararon que habían pacientes que a veces no asistían a las consultas por razones económicas y de transporte, que con fre- 
cuencia les dificultaba el traslado al servicio, ya que el servicio de salud no contaba con vehículo. Por lo tanto, algunos de los inspectores trataban de buscarle solución a la problemática ingeniándosela de alguna manera para llevarlos hasta el centro dispensador de salud, pero cuando éstos no podía ayudarlos el usuario simplemente perdían la consulta. Acarreando como consecuencia que el tratamiento no se le colocara como tenía que ser, sino que pasaba demasiado tiempo entre una terapia y otra, trayendo como consecuencia que la efectividad y eficacia del tratamiento no fuera la misma en comparación a otros pacientes que se les aplicaba la vacuna de manera puntual.

Los funcionarios de salud también afirmaron que cuando un paciente dejaba tiempo sin ir a la consulta y veía al funcionario en sus comunidades realizando un operativo o cualquier actividad vinculada con su trabajo, éstos adoptaban dos tipos de comportamiento: unos se escondían y los otros sencillamente se acercaban a ellos manifestándoles la causa por las cuales no acudieron al servicio. Posteriormente, el promotor social o el inspector de la salud los orientaba y motivaba para que regresaran nuevamente a la consulta.

Asimismo, explicaron que también habían pacientes que aunque no culminaban su tratamiento ya estaban curados, pues, esto se debía a su estructura inmunológica que los hacia sanar rápidamente, lo cual traía como consecuencia que desertaran del tratamiento sin todavía estar de alta, porque creían que ya no les hacia falta. No obstante, estaba el otro extremo de pacientes que aunque ya habían recibido toda la terapia de vacuna- ción aún no estaban curados, esto se debía a la diferencia de su estado inmunológico, y además, por no tener una buena asepsia de sus lesiones.

De igual modo, expusieron que para ellos era sumamente incomodo el hecho de que los pacientes acudieran al Servicio en busca de su tratamiento y que en ocasiones por diversas razones, ellos no pudieran administrarle los medicamentos, o en caso contrario que hubiera el tratamiento en cantidades reducidas pero que solamente asistiera un paciente. Cuando esto último sucede según ellos se corre el riesgo de perder el resto del contenido del frasco de la vacuna porque tiene muy poco tiempo de duración, pero se le aplica el tratamiento al enfermo o sino se le cita para que regrese otro día. Por esa razón, expresaron que era mejor la forma como elaboraba anteriormente la vacuna, ya que venía cada frasco en dosis única.

En relación con la prevención manifestaron que continuamente en las consultas de control se les suministraba información a los pacientes y a sus acompañantes sobre la enfermedad, las formas de contraerla, los modos preventivos y como se debían curar las lesiones ulcerativas. Sin embargo, como éstos en su mayoría sabían que al recibir la inmunoterapia quedaban inmunizados no ponían en práctica dichos modos preventivos. De igual modo, otros aun cuando se explicaba la importancia que tenía la cura o limpieza de las ulceras para hacer más efectivo el tratamiento hacían caso omiso a las orientaciones que al respecto se les daban. Con frecuencia este tipo de pacientes regresaban a la consulta con las lesiones leishmánicas infectadas y hasta 
más grandes, debido al descuido y su propia negligencia.

En cuanto al fenómeno salud-enfermedad, los pacientes manifestaron que la salud para ellos era algo importante y significaba estar bien por no tener ningún tipo de dolencia, malestar o enfermedad que les impidiera realizar de forma habitual sus tareas cotidianas en el conuco y su hogar; además, que explicaron que cuando se enfermaban no podían ir al conuco a realizar su trabajo como de costumbre, ya que las dolencias, padecimientos y malestares se lo impedían, lo cual trae como consecuencias que descuidaran su trabajo (no preparar el conuco para la siembra y no poder recoger los productos cosechados), lo que traía como resultados que la cosecha se perdiera y que se pusiera en peligro la fuente principal de ingresos del grupo familiar, ya que mayoría de éstos son agricultores que viven de sus cultivos. Por tal motivo, expusieron que enfermarse para ellos era algo malo o mortal por el sólo hecho no poder realizar sus actividades domésticas y de trabajo como lo hacían de manera habitual. Además, que muchos de carecer de los recursos necesarios para comprar medicinas y sobre todo no contaban con los medios como trasladarse hacia los centros dispensadores de salud.

\section{Consideraciones finales}

Actualmente, los problemas de la salud en su mayoría, están relacionados con los hábitos y prácticas cotidianas de las personas con entidades biológicas específicas, donde las modificaciones de los patrones de conducta de la gente, no son fáciles de lograr. En ese sentido, el estilo de vida constituye significativamen- te el estado salud, por referirse a todas decisiones individuales, sobre todo aquellas en las que los sujetos tienen cierto grado de control y que pueden afectar su salud positiva o negativamente. Esta situación aunada a la percepción que tienen las personas en torno al fenómeno salud-enfermedad, especialmente las que padecen la Leishmaniasis; $y$ en torno a la gestión del Servicio de Dermatología Sanitaria; de alguna manera u otra contribuyen a la prevalencia y reemergencia de la endemia en la zona estudiada.

Para disminuir el incremento y controlar la Leishmaniasis en el estado Sucre, es preciso que en primer lugar, se generen condiciones necesarias (acondicionamiento de una infraestructura, incremento del personal técnico-operativo y dotación de equipos para el mantenimiento de medicamentos, trasportes, y otros), así como efectuar todas las actividades y tomas de decisiones pertinentes para lograr la descentralización de la atención, tratamiento y control de la enfermedad. El punto de partida debe ser el Servicio de Dermatología Sanitaria; ya que, allí es donde se encuentran concentradas todas las acciones administrativas y operativas para llevar a cabo las prácticas curativas de esta endemia. De esta manera, el servicio sería más eficaz y efectivo, porque estaría más cerca de las comunidades afectadas y así se garantizaría el acceso de un mayor número de pacientes que por diversas razones (socio-económicas, culturales, falta de transporte o de información) no acuden a las consultas.

Otro aspecto clave, es que los prestadores del servicio orienten, eduquen y motiven a los pacientes sobre la enfermedad; y además, los incorporen en 
programas comunitarios de salud para que ellos puedan participar activamente en función de la prevención de la enfermedad desde sus respectivas comunidades; dejando claro lo fundamental que representa el hecho de que el paciente tiene que tomar muy en cuenta que la asepsia o limpieza de las lesiones o ulceraciones conllevan a la mayor efectividad del tratamiento.

Por otra parte, se deduce que la mayoría de los pacientes, no pacientes e informantes clave de este estudio tienen una imagen muy particular de la Leishmaniasis, generalmente la ven como una llaga o úlcera, que no merece mucha importancia, no otorgándosele el status de enfermedad. Algunas veces, las causas de esta endemia son atribuidas a un acto de brujería o a un castigo divino; de allí que, para el tratamiento recurren a los llamados curanderos o "botánicos". Aunque otras veces también se apela a éstos últimos por otras razones, relacionadas con las creencias populares, la escasez de recursos económicos o la facilidades de acceso al tratamiento que brindan estos curanderos. Mientras, que para otro grupo de personas la Leishmaniasis es considerada una llaga o úlcera, le dan la connotación de enfermedad, buscan los modos curativos para curarse acudiendo al servicio médico más cercano, para el diagnóstico y tratamiento de la endemia. Se detectó también un grupo de pacientes que asumieron una actitud diferente; pues, aunque asistieron al SDS y resultaron positivo en el diagnóstico, se volvieron renuentes y no asistieron más a la consulta del control regular de la endemia. Este tipo de pacientes prefiere efectuarse su propio tratamiento casero sin importarles la forma ni las condiciones como lo hacían y menos lo que se ponían en la úlcera, incurriendo de este modo en las prácticas erróneas que han traído consecuentemente la prevalencia y reemergencia de la endemia en estas zonas de estudio.

En tal sentido, es conveniente señalar que la información que poseen los habitantes de las áreas de estudio o usuarios respecto al servicio que presta el SDS es todavía insuficiente, debido a que las personas que a pesar de que tienen conocimiento de la patología, en su mayoría desconocen los modos preventivos de la misma, aunque el personal técnico de dicho servicio ha venido realizando su trabajo con los pacientes que acuden a las consultas del control regular de la enfermedad. Hay otro grupo de pacientes que aún teniendo la información en torno a las formas de prevención de la Leishmaniasis no las ponen en práctica. Muchos de ellos se acostumbraron a vivir con la endemia y la asumen como un aspecto más de su vida cotidiana, que no representa ningún problema.

En cuanto a la gestión que realiza el Servicio de Dermatología Sanitaria, esta se considera positiva debido a que los pacientes perciben que el equipo de salud cumple a cabalidad de manera puntual, responsable, con mística de servicio y vocación las diferentes actividades que comprenden el desempeño de su trabajo. Además, de las diversas diligencias que involucran la gerencia de ese servicio, el cual se evidencia en la labor fundamental y rol central desempeñado en este organismo en la ejecución de las diferentes acciones con respecto a la atención, tratamiento y control de las Leishmaniasis. 
Asimismo, la investigación arrojó fundamentalmente la necesidad de tomar en consideración las características socio-demográficas y socio-culturales de los habitantes de las localidades en estudio. Estas son áreas rurales, endémicas, desprovistas de servicios sanitarios y una adecuada estructura vial. El estilo de vida de los pobladores posibilita las emergencias y reemergencias de la Leishmaniasis, debido a que la mayoría de sus habitantes son agricultores que viven de sus cultivos y no poseen los recursos económicos para trasladarse hasta las zonas urbanas donde funcionan los centros dispensadores de salud. En este sentido, es evidente que tales restricciones conllevan a que se deterioren cada día más las condiciones de vida de esas personas; y por supuesto, que exista un mayor riesgo de contraer o perpetuar la enfermedad. Por esa razón, se hace necesario tomar en consideración la estrategia de descentralización del tratamiento por lo menos por municipio y crear las condiciones adecuadas, para que se puedan eliminar las contradicciones o debilidades del Servicio.

\section{Referencias Bibliográficas}

Aranda, Pastor (1991), Epidemiología General. Mérida, Concejo de Publicaciones Universidad de los Andes.

Berger, Peter y Luckman, Thomas (1994), La Construcción Social de la Realidad. Amorrortu Editores. Buenos Aires.

Coordinación Regional de Dermatología Sanitaria $(\mathrm{S} / \mathrm{F})$, Estrategias Metodológicas de Promoción en Salud Hacia un Modelo de Atención para el Diagnóstico, Tratamiento, Control y Prevención de la Leishmaniasis en el estado Sucre. Estado Sucre.

Kornblit, Ana (2000), Las Representaciones Sociales Acerca de la Salud y la Enfermedad: una puesta al día. En BRICEÑO, Roberto; de Souza María y Coimbra, Carlos E. (Coord.). Salud y Equidad: una mirada desde las Ciencias Sociales. Edit. Fiocruz, Río de Janeiro. Brasil.

Luna, Yvelisse del Jesús (2001), Estudio Restrospectivo y Prospectivo de la Seroprevalencia de la Leishmaniasis en la Región Paria. Estado Sucre.

Molina, María y Morera, Nidia (1999), La Gerencia de los Servicios Sociales. Editado por CUMEN/Humanitas, Argentina.

Moscovici, Serge (1961), La Psychanalyse, son image et son Public. Paris. Put.

OMS (2002), Situación de las Enfermedades Trasmisibles: Leishmaniasis. Disponible en: http:/www.who.int/cuac/ dises/Leish/index.html. 